\title{
Generación de Spray por Vibración de un Fluido Viscoelastico
}

\author{
Gabriel Ferreyra $^{1}$, Sonia Lustig ${ }^{1}$, Marta Rosen $^{1^{*}}$ y Cecilia Cabeza ${ }^{2}$ \\ (1) Universidad de Buenos Aires, Facultad de Ingeniería, Grupo de Medios Porosos, \\ Paseo Colón 850, $2^{\circ}$ piso, 1063 Buenos Aires-Argentina (e-mail: mrosen@fiuba.edu.ar) \\ (2) Universidad de la República, Facultad de Ciencias, Insituto de Física, Iguá 4225, \\ Montevideo-Uruguay \\ *autor a quien debe ser dirigida la correspondencia
}

\section{Resumen}

En este trabajo se estudia la emisión de spray de liquidos viscoelasticos generada mediante vibración, asi como la influencia de sus propiedades físicas en el tamaño de las gotas. El recipiente conteniendo el fluido es sometido a un campo de fuerzas vertical periódico, del cual se controlan la amplitud de la excitación y la frecuencia del mismo. Se utilizaron diferentes mezclas de queroseno con agregado de polímero viscoelástico, resultando validos los modelos aplicados a fluidos Newtonianos dentro de las condiciones de trabajo. Tambien se obtuvo la dependencia del tamaño de gota con la cantidad de polimero agregado, la aceleracion y la frecuencia aplicadas. Se concluye que los modelos desarrollados para fluidos Newtonianos pueden ser aplicados a liquidos de características similares a los utilizados en estas experiencias.

Palabras clave: fluidos viscoelásticos, formación de spray, generacion de gotas, fluidos newtonianos

\section{Spray Generation by Vibration of a Viscoelastic Fluid}

\begin{abstract}
In this paper, viscoelastic liquids spray emission generated by vibration and the influence of their physical properties are studied. The fluid contained in a cell is subjected to a field of periodic vertical forces, for which the excitation amplitude and frequency are controlled. Different mixtures of kerosene with viscoelastic polymer were used and results were modelled using equations applicable to Newtonian fluids with acceptable results within the values of the operating conditions considered. Also, the dependence of drop size with the quantity of polymer added, the acceleration and the frequency applied was determined. It is concluded that Newtonian fluids models can be applied to liquids with characteristics similar to those studied in this work.
\end{abstract}

Keywords: viscoelastics fluids, spray formation, drop generation, newtonian fluids 


\section{INTRODUCCIÓN}

El fenómeno de la formación de estructuras, digitación y producción de spray en la superficie libre de un fluido sometido a una vibración vertical periódica, conocido como Inestabilidad de Faraday (Kumar, 1999; Cabeza et al., 2006). ha sido objeto de un amplio estudio en la última década, transformándose en una de las experiencias emblemáticas en el estudio de inestabilidades hidrodinámicas debido a la sencillez del montaje experimental.

Cuando una capa de fluido es excitada utilizando un campo de fuerzas paralelo al campo gravitatorio, se forman en la superficie libre estructuras espacio-temporales que dependen de la viscosidad, tensión superficial, profundidad de la capa de fluido y la frecuencia de excitación. A partir de un cierto umbral de excitación, se produce una bifurcación sub-crítica y el sistema evoluciona al caos a través de una ruta de duplicación de período (Rosen et al., 2006). Para valores suficientemente altos de aceleración, se rompe la simetría de las estructuras desarrolladas y se forman los denominados "fingers", estructuras fuertemente localizadas espacialmente, a partir de los cuales y aumentando la aceleración aplicada, se producirá la eyección de gotas o spray (Cabeza et al., 2006), debido al rompimiento de la superficie libre (Yule y Suleimani, 2000; Vukasinovic et al., 2007; Villermaux, 2007).

Esta experiencia tiene importantes aplicaciones desde el punto de vista tecnológico (Goodrige et al., 1997; Pyrtle, 2005) por ejemplo permite analizar las etapas que llevan a la producción de spray en sistemas de alimentación de combustible para motores y turbinas, ya que el control de la distribución de tamaños de las gotas de combustible es una herramienta importante para ajustar la combustión y emisión de gases contaminantes (CO, NOx). La distribución de tamaños de gotas dentro de un spray es determinante en los tiempos de combustión de las mismas y por ende, su influencia en los procesos termoquímicos que se ponen en juego. El propósito de la atomización de combustibles en motores (Waters, 2000) y quemadores es incrementar la relación superficie/volumen del líquido, para de esta manera aumentar el área de contacto entre el combustible y el aire, favoreciendo la evaporación del combustible y las reacciones de la combustión (Valtadoros et al., 1991) En forma convencional, la atomización se produce por medios mecánicos o neumáticos. En los últimos años se han desarrollado trabajos sobre la influencia de los polímeros de alto peso molecular y determinadas características reológicas en la distribución de tamaños de gotas. Un campo de aplicación en desarrollo es también el aprovechamiento de spray para refrigeración de superficies, tales como equipos electrónicos de alta potencia o enfriamiento rápido de materiales.

El presente trabajo está focalizado en el proceso de generación de spray, en el umbral de eyección y en el tamaño de las gotas, para un fluido que es utilizado como modelo de hidrocarburo con el agregado de un polímero viscoelastico (Ferguson et al., 1992). Si bien se han puesto a punto distintas técnicas de obtención de imágenes para estudiar el proceso que se produce a altas velocidades (Thoroddsen et al., 2008), en este caso, se describe el sitema experimental que ha permitido una precisa adquisición de imágenes y determinación de diametros. Experiencias similares fueron realizadas con soluciones de óxido de polyetileno, pero en este ultimo trabajo, lo que se modificó fue la composición del solvente (Mun et al., 1998).

En una primera etapa se validan los resultados obtenidos para el umbral de eyección de gotas, comparándolos con resultados previos en fluidos Newtonianos de baja viscosidad. En una segunda etapa se analizan los resultados de la distribución de tamaños de gotas en función de la cantidad de polímero adicionado al fluido base, variando los parámetros de control de la experiencia: aceleración y frecuencia.

\section{DISPOSITIVO EXPERIMENTAL}

El dispositivo experimental utilizado es mostrado en la figura 1. Este tipo de montaje es muy robusto ya que permite un sencillo y preciso control de los parámetros del sistema, pudiéndose reproducir con total precisión los resultados experimentales. El fluido es colocado dentro de una celda de acrílico, de dimensiones 100×100x60 mm, unida rígidamente al vibrador, que es excitado mediante un generador de funciones, previa etapa de amplificación. La aceleración fue medida indirectamente 
a través de un acelerómetro (ADXL 150), cuyo rango de trabajo es de 100 g p.a.p. máximo, con un error de $\pm 2 \%$. El tipo de régimen, armónico o sub-armónico, que puede presentar el sistema, depende de la altura de la capa de fluido, por lo cual ésta se mantuvo constante a lo largo de todas las experiencias en $\mathrm{h}=4 \mathrm{~mm}$, garantizando el desarrollo de un régimen sub-armónico. Además los resultados presentados en este trabajo fueron realizados bajo las hipótesis de aguas poco profundas ya que se trabajó en el límite $\mathrm{kh} \approx 1$, siendo $\mathrm{k}$ el número de onda y $\mathrm{h}$ la profundidad de la capa de fluido. Así mismo, el rango en frecuencia garantizó que en toda la experiencia se trabajó en el régimen capilar, ya que la frecuencia de corte entre el régimen gravitatorio y el capilar es del orden de $10 \mathrm{~Hz}$.

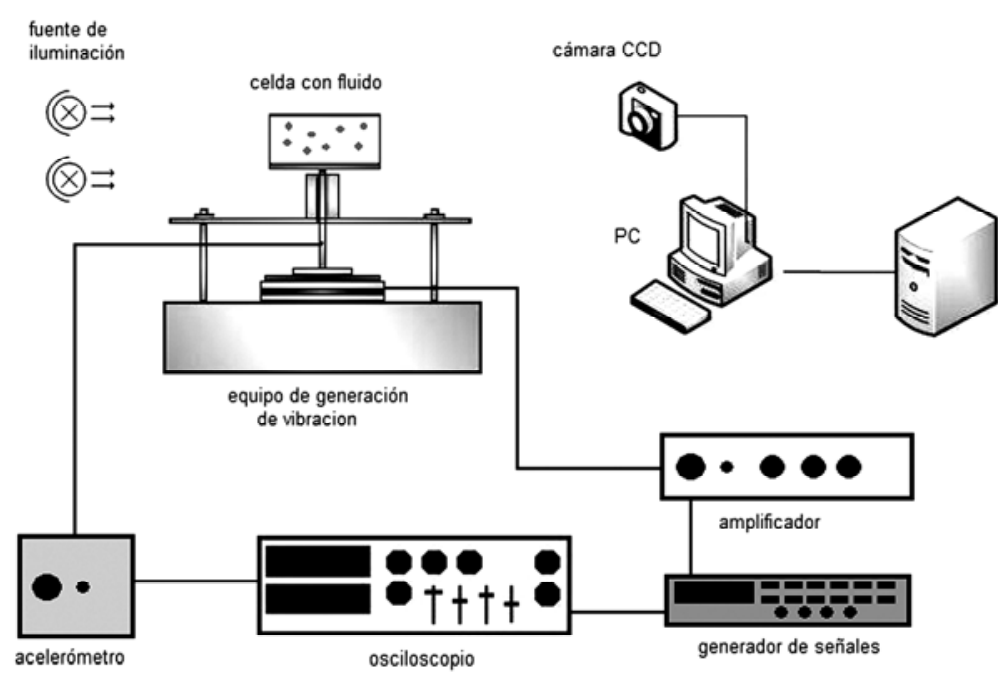

Fig. 1: Esquema del dispositivo experimental.

El rango de frecuencia para el cual se estudió el umbral de eyección varió entre 50 y $200 \mathrm{~Hz}$ y las aceleraciones respectivas entre 5 y $25 \mathrm{~g}$ pico a pico. Para los tamaños de gotas, se realizó un estudio estadístico de promedios en función de frecuencia, aceleración y porcentaje de polímero agregado. El rango de trabajo correspondió de 200 a $300 \mathrm{~Hz}$ y desde $30 \mathrm{~g}$ pico a pico hasta $80 \mathrm{~g}$ pico a pico.

El umbral pudo ser detectado visualmente con la cámara CCD. El criterio para determinar el momento de eyección ha sido la aparición de dos gotas durante periodos de diez segundos.

En este trabajo se utilizaron diversas mezclas de Hidrocarburo (Queroseno Comercial) con un fluido viscoelástico de alto peso molecular (PIB 6500 ppm), en diferentes proporciones. Se analizó el comportamiento para el fluido puro, y para concentraciones de polímero que variaron entre $0.1 \%$ y $2 \%$. La viscosidad de las muestras fue medida utilizando un reómetro (Physica MCR 300 rheometer). Este análisis mostró que todas las mezclas utilizadas tienen una respuesta newtoniana para el rango de frecuencias de trabajo, ya que la viscosidad se mantiene constante al variar el esfuerzo de corte, pero cambia su valor absoluto en función de la cantidad de polímero adicionado. Asi los valores de viscosidades obtenidos para las soluciones con distintas concentraciones de polímero son: PIB $2 \%$ 2.8 cP; PIB 1\% 2,5 cP; PIB 0,5\% 2,2 cP; PIB 0,3\% 2 cP; PIB 0,2\% 1,9 cP respectivamente.

Con el fin de obtener buenas imágenes, la celda fue iluminada con luz indirecta y se utilizó una cámara CCD a $200 \mathrm{fps}$ para registrarlas. En la figura 2 se muestra una imagen de las gotas generadas en el caso de la mezcla de queroseno adicionado con $0.5 \%$ de PIB, a una frecuencia de $250 \mathrm{~Hz}$ y una aceleración de $79 \mathrm{~g}$.

Para estudiar la generación de "spray", se realizaron videos y a partir de los mismos se realizó un análisis de tamaños promedio de gotas en función de frecuencia, aceleración y porcentaje de polímero agregado. 


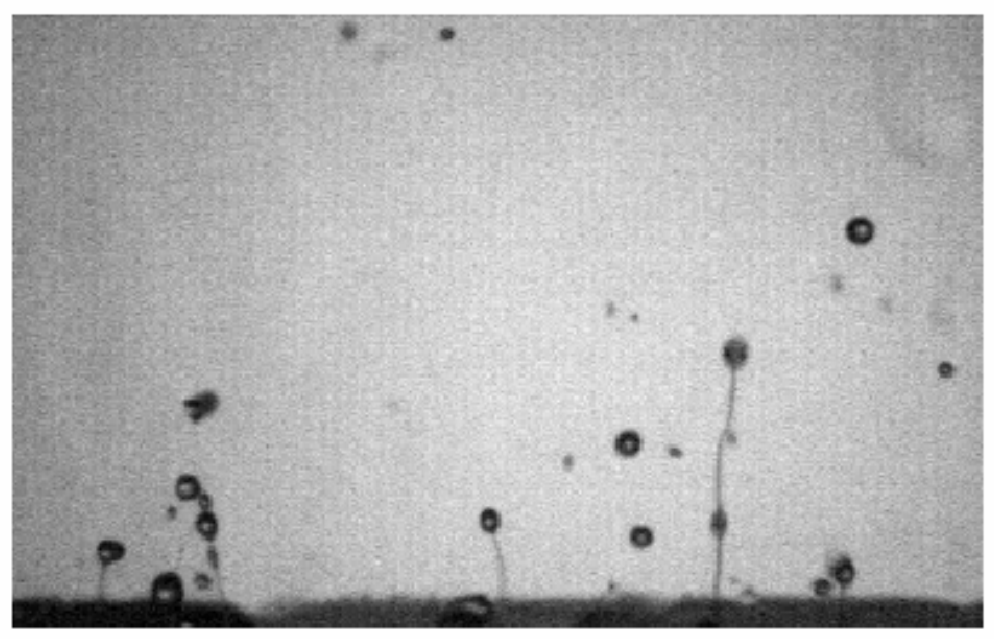

Fig. 2: Eyección de gotas en el caso $0.5 \% \mathrm{PIB}$, frecuencia de $250 \mathrm{~Hz}$.

\section{RESULTADOS Y DISCUSIÓN}

En este punto se analizan los parámetros principales considerados con el fin de caracterizar el sistema en estudio.

Umbral de eyección

Como forma de validar los resultados obtenidos para el umbral de eyección de gotas, se utilizó el modelo propuesto en trabajos previos por Goodridge et al. (1996), quienes presentan la siguiente adimensionalización para la aceleración y la frecuencia, obtenida a partir de argumentos empíricos:

$$
\begin{aligned}
& \omega^{*}=\frac{\omega_{0} v^{3}}{(\sigma / \rho)^{2}} \\
& a^{*}=\frac{a v^{4}}{(\sigma / \rho)^{3}}
\end{aligned}
$$

Donde $\omega_{o}$ es la frecuencia de excitación, $v$ la viscosidad cinemática, $\sigma$ la tensión superficial y $\rho$ la densidad del fluido y a la aceleracion aplicada.

A partir de estas expresiones es posible encontrar una ley de escala para el umbral de eyección. Para fluidos de baja viscosidad se obtiene:

$a^{*} \propto \omega^{4 / 3}$

En la figura 3 se representa la aceleración umbral en función de la frecuencia de excitación, para todas las mezclas utilizadas.

En la figura 4 se muestra la curva de las variables adimensionalizadas definidas en la ec. 1. Se ha agregado también como referencia la curva umbral del agua, con el objetivo de mostrar que el escalamiento realizado se ajusta correctamente. Puede verse que el ajuste realizado según la ec. 2 (curva llena) resulta adecuada dentro de esta escala de baja viscosidad con lo que los resultados obtenidos presentan una correlación satisfactoria. 


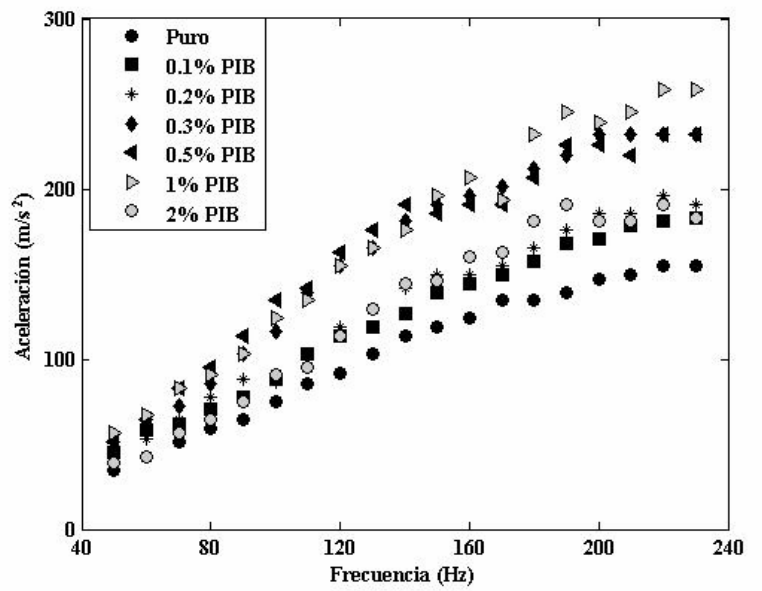

Fig. 3: Umbral de aceleración para la eyección de gotas en función de la frecuencia aplicada

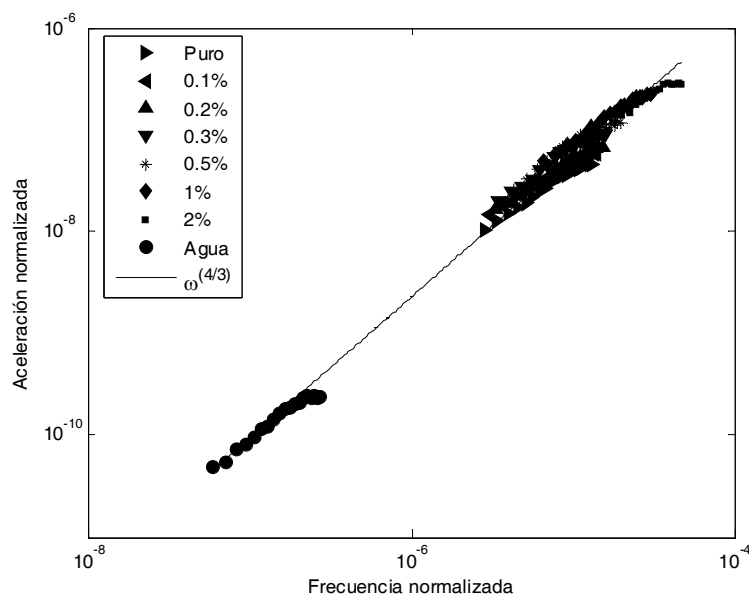

Fig. 4: Aceleración normalizada en función de la frecuencia (ec.1)

Si bien las relaciones empleadas fueron obtenidas para fluidos Newtonianos, a partir de este estudio para fluidos viscoelasticos, se pueden generalizar los resutados para liquidos que presenten una region de viscosidad constante frente a la variacion del esfuerzo de corte, como los que se presentan en este trabajo.

\section{Tamaños de gota}

En la segunda etapa de este trabajo se estudió la distribución de tamaños de las gotas en función de la cantidad de polímero adicionado. El rango de aceleración fue elegido de manera de estar por encima del umbral de eyección.

Así mismo se estudió la dispersión de tamaños que presentaban las gotas. En las figuras 5a,5b y 5c, se muestran los resultados obtenidos donde los ejes elegidos permiten comparar con las relaciones obtenidas por otros autores (Pyrtle, 2005). Puede observarse que a medida que se aumenta la aceleración, manteniendo fija la frecuencia, el diámetro de las gotas aumenta. Este comportamiento es el mismo para todas las muestras estudiadas. Sin embargo, es posible notar que la influencia del agregado de PIB se evidencia especialmente a bajas frecuencias con un aumento del tamaño promedio.

A medida que la frecuencia aumenta, la influencia del agregado de polímero se hace menos notoria hasta que para la frecuencia de $275 \mathrm{~Hz}$, el porcentaje de polímero agregado no tiene prácticamente 
efecto. Hay que resaltar también de que con el aumento de la frecuencia el tamaño promedio de gota disminuye independientemente de la aceleración.

Con el fin de estudiar la dispersión en el tamaño de las gotas ya que el mismo no muestra uniformidad en cada experiencia, se calculó la desviación estándar en los tamaños de las mismas. La distribución obtenida está fuertemente vinculada a la etapa previa de la eyección, donde la estructura de los "fingers" a partir de los cuales se produce el rompimiento de la superficie libre condiciona el tamaño final de la gota.

Un resultado importante es el hecho de que el agregado de hasta un $0,5 \%$ de PIB en el fluido base se reduce la desviación estándar de tamaños en aproximadamente un $20 \%$, lo que permite un control sobre la homogenización del spray.
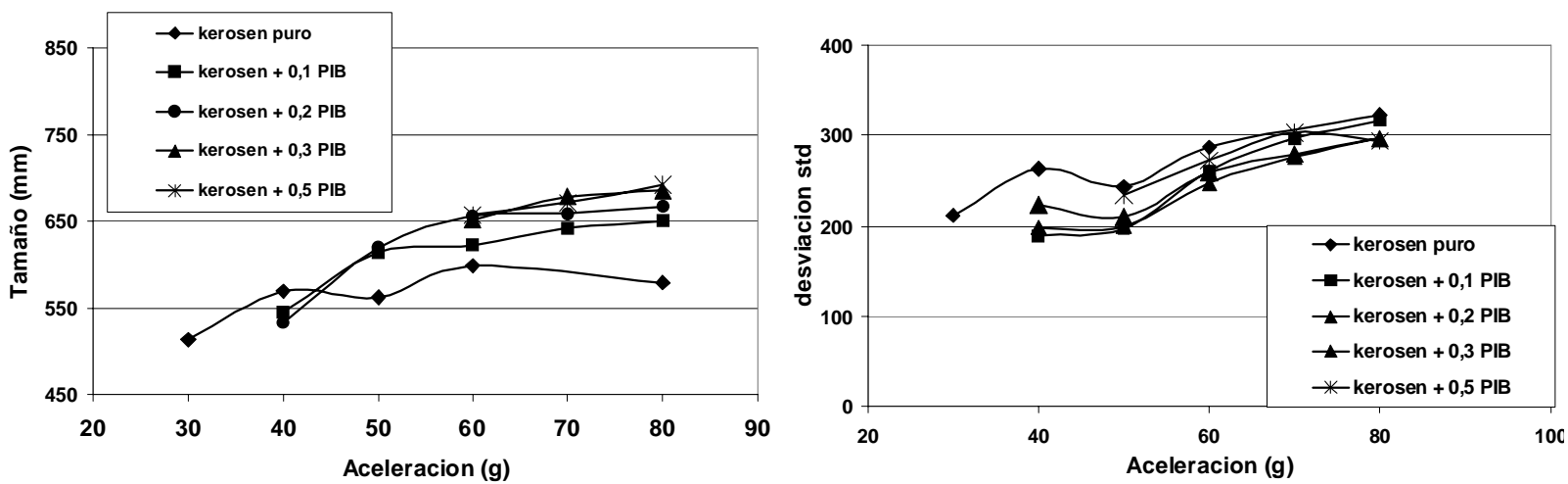

(a)
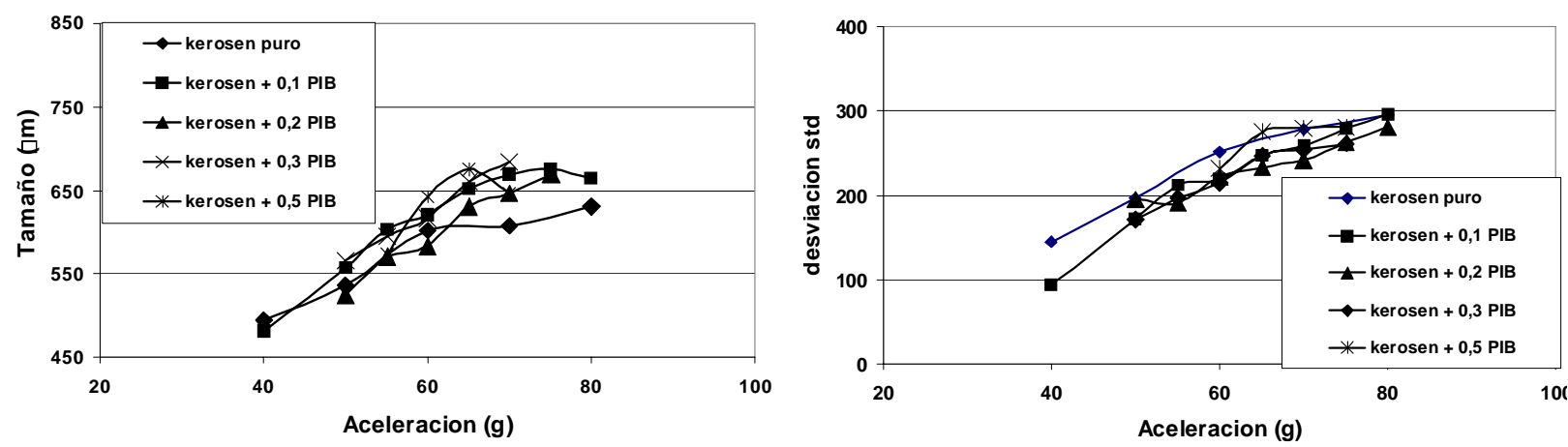

(b)
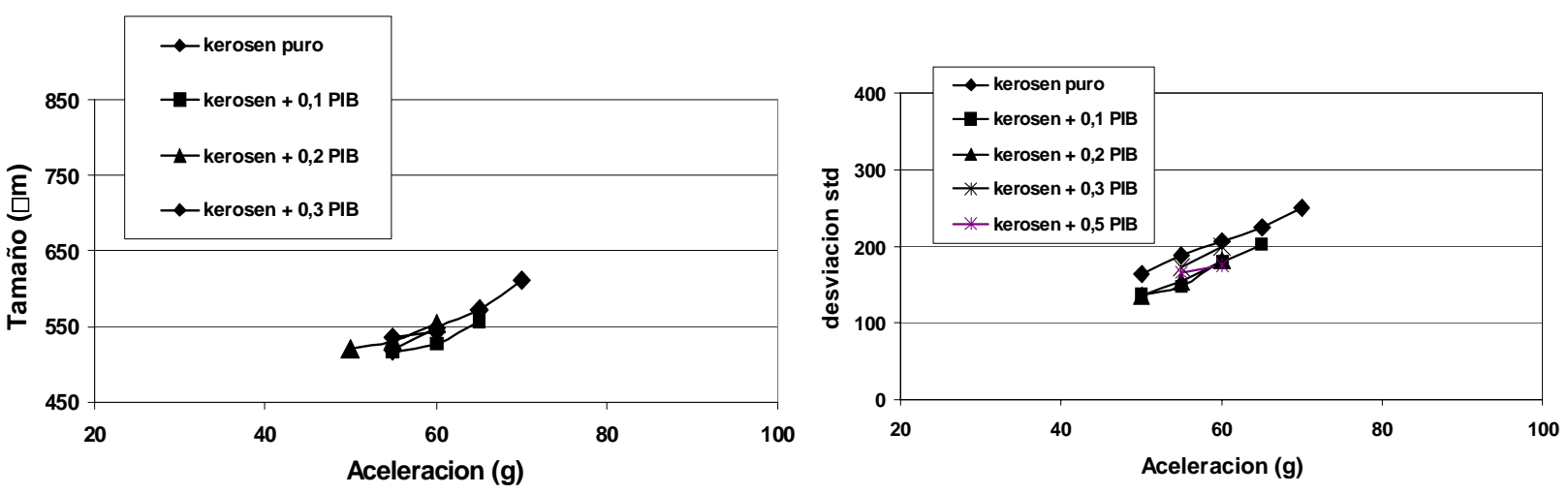

(c)

Fig. 5: Tamaño de gotas y desviación estándar en función de la aceleración. (a) f = $225 \mathrm{~Hz}$; (b) $\mathrm{f}=259 \mathrm{~Hz}$; (c) $\mathrm{f}=275 \mathrm{~Hz}$ 


\section{CONCLUSIONES}

Los resultados presentados en este trabajo muestran que los modelos desarrollados para fluidos Newtonianos pueden ser aplicados a liquidos de características similares a los utilizados en estas experiencias: rango de viscosidades bajas y constantes. Se ha obtenido buena concordancia en el umbral de eyección.

Por otro lado, el tamaño promedio de las gotas del "spray" presenta una clara dependencia con la cantidad de polímero agregado. A medida que se aumenta la concentración del mismo, el tamaño promedio aumenta, y la dispersión de resultados resulta menor.

Así mismo se ha observado que con el aumento de la aceleración y para una frecuencia dada, el tamaño de gota muestra una clara tendencia a incrementarse. Mientras que, respecto a la frecuencia, disminuye cuando ésta aumenta.

Este es un tema en estudio con una importante perspectiva industrial siendo el interés de este grupo extender el estudio al rango de las frecuencias altas.

\section{AGRADECIMIENTOS}

Al Proyecto PEDEICBA, URU/84/002/UNDP, al CLAF y al Subsidio 1041 de Universidad de Buenos Aires.

\section{REFERENCIAS}

Cabeza, C., M. Rosen, G. Ferreyra y G. Bongiovanni; Dynamical behavior of digitations state in Faraday waves with a viscoelastic fluid, Physica A : 371(1), 54-58, (2006).

Ferguson, J., N.E Hudson y B.C.H. Warren; The break-up of fluids in an extensional flow field, Journal of Non-Newtonian Fluid Mechanics: 44(1), 37-54 (1992).

Goodridge, C.L., W.T. Shi, H.G.E. Hentschel y D.P. Lathrop; Viscous effects in droplet-ejecting capillary waves, Physical Review Letters: 56(1), 472-475 (1997).

Goodridge, C.L., W.T. Shi y D.P. Lathrop; Threshold Dynamics of Singular Gravity-Capillary Waves, Physical Review Letters: 76(11), 1824-1827 (1996).

Kumar, S.; Parametrically driven surface waves in viscoelastic liquids, Physics of Fluids: 11(8), 1970 (1999).

Mun, R., J.A. Byars y V. Boger; The effects of polymer concentration and molecular weight on the breakup of laminar capillary jets, Journal of Non-Newtonian Fluid Mechanics: 74(1-3), 285-297 (1998).

Pyrtle, F.; Vibration Induced Droplet Generation From A Liquid Layer For Evaporative Cooling In a Heat Transfer Cel , Ph.D. Thesis, Georgia Institute of Technology (2005).

Rosen, M., M. Piacquadio, C. Cabeza y C. Lizarralde; Multifractal spectra in the Faraday experiments, Proceedings, The 9th. Experimental Chaos Conference-ECC9, Sao jose dos Campo, Brasil, 29 de Mayo al 1 de Junio (2006).

Thoroddsen, S.T., T.G. Etoh y K. Takehara; High-speed Imaging of Drops and Bubbles, Annual Review of Fluid Mechanics: 40(1) , 257-285, (2008).

Valtadoros, T.H., V.W. Wong y J.B. Heywood; Fuel Additive Effects on Deposit Build-Up and Engine Operating Characteristics, ACS Preprints: 36(1), Symposium on Fuel Composition/Deposit Formation 
Tendencies, Division of Petroleum Chemistry, American Chemical Society, Atlanta, April 14-19 (1991).

Villermaux, E.; Fragmentation, Annual Review of Fluid Mechanics: 39(1), 419-446 (2007)

Vukasinovic, B., M.K. Smith y A. Glezer; Mechanisms of free-surface breakup in vibration-induced liquid atomization, Physics of Fluids: 19(1) (2007).

Waters, P.; Global Warming Reduction by Polymers in Automotive Fuels, Polymer: 41(2), 1807-1808 (2000)

Yule, A. J. e Y. Suleimani; On droplet formation from capillary waves on a vibrating surface, Proc. R. Soc. Lond A : 456(1997), 1069-1085 (2000). 